\title{
La découverte d'Asplenium ruta-muraria L. subsp. ruta-muraria dans la région de Tlemcen (Algérie occidentale).
}

\author{
Brahim Babali', Abdelkader Babali, Boumediene Medjahdi ${ }^{2}$ et Mohammed Bouazza ${ }^{1}$ \\ 'Laboratoire d'écologie et gestion des écosystèmes naturels, département d’Ecologie et environnement. Université de \\ Tlemcen \\ 'Laboratoire de Gestion Conservatoire de l'eau, du Sol et des Forêts et Développement durable des zones montagneuses \\ de la région de Tlemcen. Université de Tlemcen.
}

\author{
Correspondencia \\ B. Babali \\ e-mail: miharb_babali@hotmail.fr \\ Recibido: 7 mayo 2018 \\ Aceptado: 24 septiembre 2018 \\ Publicado on-line: octubre 2018
}

\begin{abstract}
Asplenium ruta-muraria $L$. subsp. ruta-muraria a new record for Tlemcen region (Western Algeria)
\end{abstract}

Mots clés: Asplenium, chorologie, Région de Tlemcen, Algérie.

Key words: Asplenium, distribution, rare species, Tlemcen, Algeria.
Le genre Asplenium est un genre subcosmopolite, ayant le plus grand nombre d'espèces de la famille, soit près de 700 dans le monde. II est plus abondant dans les régions tropicales et subtropicales (Prelli, 2001, Bosser et al. 2008). Ce genre comporte 22 espèces dans la région Méditerranéen dont 10 sont représentées en l'Algérie (Quézel et Santa, 1962 ; Meddour, 2007). Parmi lesquelles la Rue des murailles

\section{Asplenium ruta-muraria L. subsp. ruta-muraria}

Cette ptéridophyte, tout comme de nombreuses espèces de lichens, elle est considérée comme un bio-indicateur très sensible à la pollution atmosphérique. Elle a donc, fortement régressée des grands centres urbains ces dernières années. Sa présence est souvent le signe d'une bonne qualité de l'air. (Prelli, 2001)

Cette petite ptéridophyte de quelques centimètres de haut (de $5-10 \mathrm{~cm}$ ), se trouve principalement dans les fissures de rochers calcaire et siliceux depuis le littoral jusqu'aux hautes montagnes (3200 m), dans les sites ensoleillés ou ombragés (Maire 1952). Ce taxon de distribution Circum-boréal est présent en Europe, en Asie septentrionale et occidentale jusqu'à l'Himalaya et en Amérique septentrionale. II n'est présent en Afrique du nord qu'au Maroc et en Algérie.

En Algérie l'Asplenium ruta-muraria L. est représenté par la sous-espèce ruta-muraria (Prelli, 2001 ; Meddour, 2007; Dobignard et Chatelain 2010).

C'est une plante assez rare qui ne se trouve que dans 6 secteurs des 15 secteurs biogéographiques de l'Algérie du nord mis en évidence par Quèzel et santa (1962) : K1, K3 (mais elle n'a pas été revue à la Calle), C1, AS2, AS3 (Battandier et Trabut, 1902 ; Maire, 1952 ; Quézel et Santa, 1962 ; Meddour, 2007). Elle a été signalée enfin en Oranie dans I'O1, par Santa (1949) à El-Ançor et dans la forêt de Msila par Doumergue (Herbier de Doumergue au Musée d' Oran).

Ainsi ce taxon n'a jamais été signalé dans les Monts de Tlemcen (O3), ni dans les anciennes flores algériennes (Maire, 1952 ; Quézel et Santa, 1962), ni dans les travaux sur les ptéridophytes algérienne (Meddour, 2007 ; Medjahdi et al., 2013 ; Hamel et al., 2017 a, b).

Asplenium ruta-muraria subsp. ruta-murariaest donc présent dans le secteur (O3) plus précisément dans le Monts de Tlemcen. La première station se trouve à Ghar-roubane au lieu-dit $\mathrm{Dj}$. Tachleat à $1200 \mathrm{~m}$ d'altitude; sur une pierre de géomètre $\mathrm{n}^{\circ} \mathrm{C} 6$ où il y avait deux individus (figure 1). Cette observation a été faite le 27 mars 2015. Le 25 Janvier 2018, nous avons trouvé 4 individus bien venant dans une autre station à Beni-Snous au lieu-dit Sidi Otsmane ; à $1300 \mathrm{~m}$ d'altitude (figure 1). Cette fois l'espèce a été observée sur des roches calcaires humides, où elle s'associée avec une végétation hygrophiles (ptéridophyte et spermaphytes): Asplenium ceterach L. subsp. ceterach, Asplenium trichomanes subsp. quadrivalens D. E. Mey. L., Adiantum capillus-veneris L., Allosorus acrosticus (Balb.) Christenh., Aristolochia baetica L., Rupicapnos africana subsp. oranensis (Pugsley) Maire, Sarcocapnos crassifolius (Desf.), Centranthus battandieri Maire , Silene andryalifolia Pomel, Stachys circinnata L'Her., Narcissus 


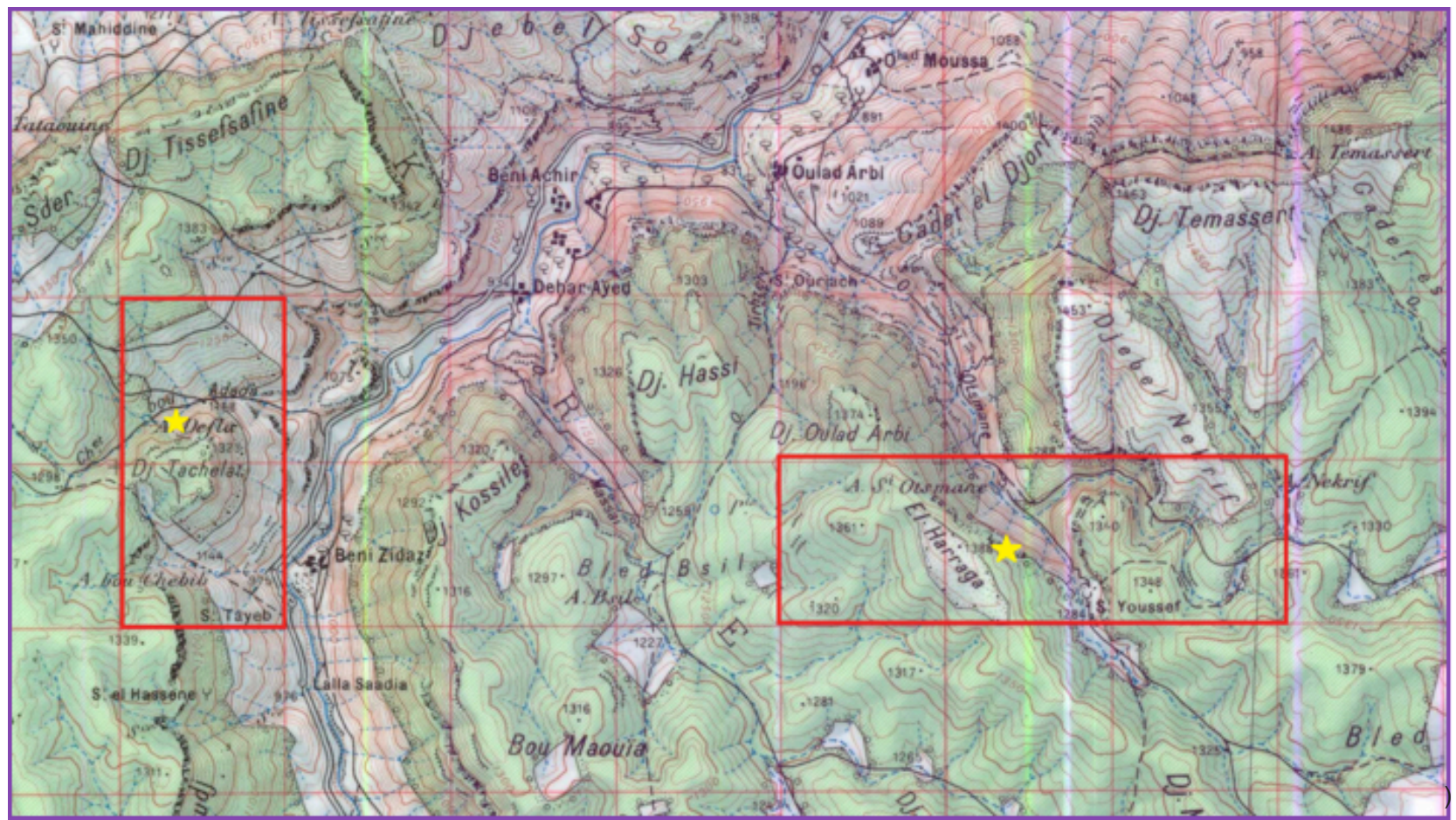

Figure 1. Stations nouvelles d'Asplenium ruta-muraria dans la région de Beni-Snous (O3). (Etoile à gauche: station de Dj. Tachleat 1200m; Etoile à droite: station de Sid. Otsmane 1300m). Source : inspiré de la carte major de Ghar-roubane (1/50000 -type 1922
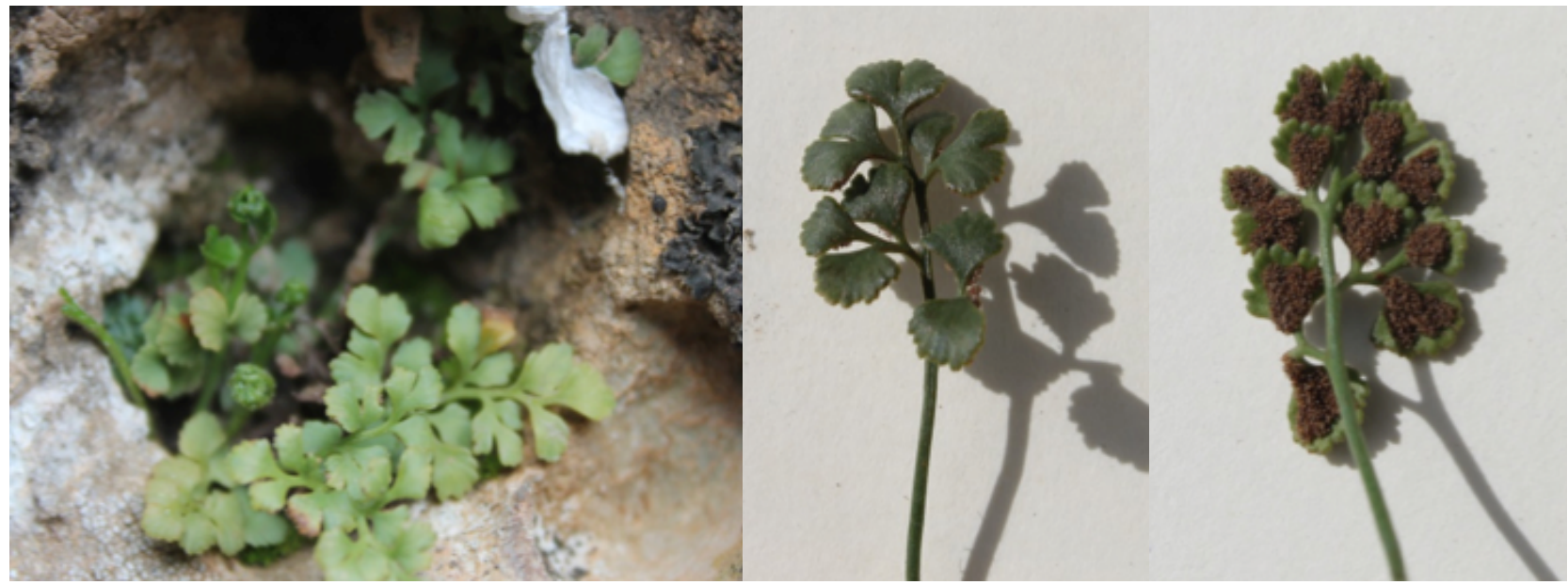

Figure 2. Asplenium ruta-muraria L. subsp. ruta-muraria (À gauche vue générale; au milieu face supérieur; à droite face inferieur de la fronde). Cliché : BABALI B., Beni-Snous le, 27 mars 2015 
cantabricus DC., Hypericum pubescens Boiss., Saxifraga globulifera Desf. subsp. globulifera, Chaenorhinum villosum (L.) Lange subsp. villosum Biscutella frutescens Coss.

Nous pouvons conclure à la fin de cette synthèse que l'Asplenium ruta-muraria subsp. rutamuraria est actuellement présent dans les secteurs biogéographiques suivant : K1, K3 ? (l'espèce est a recherché de nouveau dans ce secteur), C1, AS2, AS3. II faut ajouter les deux secteurs oranais (O3 et O1). Dans le $\mathrm{O} 3$ elle est présenté à BeniSnous et à Ghar-roubane (Monts de Tlemcen aux points GPS suivant : $34.621768,-1.590288$, Alt.:1200m ; 34.7627778, -1.4458333, Alt.:1300m). Dans le secteur oranais littoral à El-Ançor (herbier Doumergue) et la forêt de Msila (Santa, 1949). L'espèce est a recherché dans les secteurs de l'Algérois (A1 et $A 2)$. II serait très utile de créer une base de données où toutes les stations de cette espèce et des autres ptéridophytes seront géo-localisées pour étudier leur écologique et leur abondance sur le territoire national, afin d'assurer une bonne protection de ce groupe qui est en forte régression.

\section{Bibliographie}

Battandier et Trabut (1902). Flore analytique et synoptique de l'Algérie et de la Tunisie. 460P.

Bosser J., Badre F. \& Gueho J. (2008). Flore des Mascareignes, la Réunion, Maurice, Rodrigues : Ptéridophytes 1 Psilotacées à 26 Marsiléacées. Institut de recherche pour le développement, Paris, p 452.

Dobignard, A. et Chatelain, C. (2010). Index synonymique flore d'Afrique du Nord. Volume 1: Ptéridophyta, Gymnospermae et Monocotyledoneae. Editions Des Conservatoire et Jardin Botaniques, Genève. 455 p.

Hamel T., Boulemtafes A., Slimani A., El Mouaz Madoui B. et Drid M.D. (2017 a). Diversity and ecology of Pteridophytes in the Skikda region (North East Algeria). International Research Journal of Biological Sciences Vol. 6(3): 42-47.

Hamel T., Slimani A., El Mouaz Madoui B. \& Boulemtafes A. (2017 b). Pteridophytes of Edough Peninsula (North-East Algerian). Int. J. Res. Ayurveda Pharm. 8(1): 92-96.

Maire, R. (1952). Flore de l'Afrique du Nord. Volume 1. Ed. Le chevalier Paris.

Meddour, R. (2007). Taxinomie, chorologie et régression des ptéridophytes d'Algérie : synthèse bibliographique. Articles Réseaux Téla-botanica. p15

Medjahdi B., Letreuch-Belarouci A. \& Prelly R.(2013). Actualisation du catalogue des ptéridophytes du Nordouest algérien (Région de Tlemcen). Acta Botanica Malacitana, 38: 33-39.

Medjahdi B., Letreuch-Belrouci A. et Babali, B. (2017). Une Nouvelle station d'Ophioglossum lusitanicum L. en Oranie (Algérie Occidentale). Acta Botanica Malacitana, 42.

Prelli, R. (2001). Les fougères et plantes alliées de France et d'Europe occidentale.

Quézel, P. et Santa, S. (1962-Nouvelle flore de l'Algérie et des régions désertiques méridionales. CNRS, Paris, Tome 1. $558 \mathrm{p}$.

Santa, S. (1949). Catalogue des plantes de l'Algérie occidentale et du Maroc oriental. Extrait de B.S.G.A. de la Provence d'Oran. T.72, Fascicule 226, 128p. 